Original Article

Artigo Original

Weslania Viviane Do Nascimento ${ }^{1}$ Rachel De Aguiar Cassiani² Roberto Oliveira Dantas ${ }^{3}$

Descritores

Antropometria Gênero Face Estatura Adulto

Keywords

Anthropometry Gender Face Height Adult

Endereço para correspondência: Weslania Viviane do Nascimento R. Franklin Magalhães 720/33B, Vila Santa Catarina, São Paulo (SP), Brasil, CEP: 04374-000.

E-mail: fono.weslania@gmail.com

Recebido em: 22/12/2011

Aceito em: 13/9/2012

\section{Efeito do gênero, da altura corporal e da etnia nas medidas antropométricas orofaciais}

\section{Effect of gender, height and race on orofacial measurements}

Objetivo: O objetivo desta investigação foi avaliar, em população adulta, a influência do gênero, da altura corporal, e da raça nas medidas antropométricas orofaciais, e a possibilidade de correlação entre as medidas. Métodos: Foram realizadas medidas antropométricas orofaciais em voluntários assintomáticos, 56 mulheres e 44 homens, com idades entre 19 e 53 anos. As medidas realizadas foram: altura do lábio superior (sn-sto); altura do lábio inferior (sto-gn); altura do filtro (sn-ls); terço superior da face (tr-g); terço médio da face (g-sn); terço inferior da face (sn-gn); distância entre o canto externo do olho (ex) e o cheilion (ch). Resultados: Todas as medidas realizadas foram maiores nos homens do que nas mulheres. Houve correlação positiva entre a maioria das medidas realizadas. Não houve correlação entre a altura corporal e as medidas antropométricas orofaciais, exceto para a medida do terço médio da face ( $\mathrm{g}$-sn) nos homens, quando houve correlação positiva fraca. Na comparação com indivíduos da raça branca os indivíduos da raça negra tiveram maiores medidas do terço inferior da face (sn-gn) e entre o canto externo do olho (ex) e o cheilion (ch) esquerdo. Conclusão: Homens têm maiores medidas antropométricas orofaciais do que as mulheres. A altura corporal dos indivíduos parece não influenciar estas medidas.

\begin{abstract}
Purpose: The aim of this study was to assess the influence of gender, height and ethnicity on anthropometric orofacial measures in the adult population, and the possible correlation between these measures. Methods: Anthropometric orofacial measurements were performed in asymptomatic volunteers, being 56 women and 44 men, aged 19 to 53 years. The measure were: upper lip height (sn-sto); lower lip height (sto-gn); filter height (sn-ls); upper third of the face (tr-g); middle third of the face (g-sn); lower third of the face (sn-gn); and distance between the outer canthus (ex) and cheilion (ch). Results: All measurements were larger for men than for women. There was a positive correlation between most of the measures. There was no correlation between height and anthropometric orofacial measures, except for the middle third of the face ( $\mathrm{g}$-sn) of men, which had a weak positive correlation. Afro-Brazilian subjects presented larger measures for the lower third of the face (sn-gn) and for the distance between the outer canthus (ex) and the left cheilion (ch) than white subjects. Conclusion: Men have greater orofacial anthropometric measures than women. Height does not seem to have an influence on such measures.
\end{abstract}

Trabalho realizado no Departamento de Oftalmologia, Otorrinolaringologia e Cirurgia de Cabeça e Pescoço e no Departamento de Clínica Médica, Faculdade de Medicina de Ribeirão Preto, Universidade de São Paulo - FMRPUSP - Ribeirão Preto (SP), Brasil.

(1) Programa de Pós-Graduação (Doutorado) em Biociências aplicada à Clínica Médica, Faculdade de Medicina de Ribeirão Preto, Universidade de São Paulo - FMRPUSP - Ribeirão Preto (SP), Brasil.

(2) Faculdade de Medicina de Ribeirão Preto da Universidade de São Paulo - FMRPUSP - Ribeirão Preto (SP), Brasil. Fonoaudióloga do Centro Integrado de Reabilitação do Hospital Estadual de Ribeirão Preto - CIRHERP Ribeirão Preto (SP), Brasil.

(3) Departamento de Clínica Médica, Faculdade de Medicina de Ribeirão Preto, Universidade de São Paulo FMRPUSP - Ribeirão Preto (SP), Brasil.

Conflito de interesse: nada a declarar. 


\section{INTRODUÇÃO}

Diversos estudos revelam diferenças anatômicas e funcionais entre homens e mulheres em relação à boca, faringe, esfíncter esofágico superior, esôfago e deglutição ${ }^{(1-5)}$.

A antropometria oferece inúmeras vantagens no estudo da morfologia do complexo craniofacial $^{(6)}$ por utilizar técnicas simples, não invasivas, sem risco para o sujeito e com baixo custo $^{(7)}$. A literatura descreve efeitos do gênero nas medidas antropométricas orofaciais, fazendo referência a maiores medidas em homens quando comparados às mulheres ${ }^{(8,9-11)}$. Ainda, estudos revelam diferenças entre as medidas antropométricas orofaciais em diferentes etnias ${ }^{(6,8,12,13)}$.

É proposto que estas medidas são importantes para o exame fonoaudiológico, sendo cada vez mais utilizadas para avaliação, prognóstico, planejamento( ${ }^{(7)}$ e acompanhamento terapêutico. Sabe-se que a configuração das estruturas craniofaciais, bem como a musculatura orofacial e a oclusão dentária influenciam diretamente as funções de mastigação, deglutição, respiração, fala e voz ${ }^{(14)}$.

Estudo recente sobre o grau de variabilidade das medidas orofaciais entre fonoaudiólogos especialistas em motricidade orofacial demonstrou boa reprodutibilidade nas mensurações realizadas, com exceção do parâmetro de abertura da boca com a língua na papila, indicando que os dados produzidos por diferentes profissionais são confiáveis ${ }^{(15)}$.

Os objetivos desta investigação foram avaliar as diferenças entre as medidas antropométricas orofaciais de homens e mulheres adultos, a correlação entre estas medidas e a influência da altura corporal e da raça. A hipótese foi de que há influência do gênero e da altura corporal nas medidas antropométricas orofaciais.

\section{MÉTODOS}

O projeto de pesquisa foi aprovado pela Comissão de Normas Éticas do Hospital das Clínicas da Faculdade de Medicina de Ribeirão Preto da Universidade de São Paulo, processo $\mathrm{n}^{\circ} 5226 / 2008$. Todos os voluntários assinaram o Termo de Consentimento Livre e Esclarecido.

Participaram deste estudo 56 pessoas do gênero feminino, com idades entre 19 e 53 anos, média de 31,5 $\pm 10,2$ anos, e 44 pessoas do gênero masculino, com idades entre 19 e 53 anos, média de 32,4 $\pm 10,2$ anos. Foram incluídos indivíduos residentes no estado de São Paulo, de todas as classes sociais. Entre os homens, 33 eram da raça branca e 11 da raça negra, e entre as mulheres, 39 eram da raça branca e 17 da raça negra.

Foram excluídos indivíduos portadores de anomalias/alterações craniofaciais, má oclusão esquelética ou qualquer alteração anatômica que pudesse interferir nas medidas antropométricas.
Todos os sujeitos foram medidos e divididos em dois grupos: sujeitos com altura corporal variando de 1,49 a 1,65 m, média de $1,59 \pm 0,05 \mathrm{~m}$, e sujeitos que possuíam altura corporal entre 1,66 e $1,89 \mathrm{~m}$, média de $1,75 \pm 0,06 \mathrm{~m}$.

Para coletar as medidas antropométricas orofaciais utilizamos um paquímetro metálico, da marca Vonder ${ }^{\circledR}$ (Brasil). Antes da coleta das medidas foi solicitado ao voluntário que permanecesse sentado, com os pés apoiados no chão, com a cabeça em posição de repouso habitual e lábios ocluídos. Também foi solicitado que o indivíduo retirasse óculos e, após o paquímetro ser apresentado e a sua função ser esclarecida, foram realizadas as seguintes medidas: altura do lábio superior (sn-sto), que corresponde à distância entre o subnasal (sn) e o ponto mais baixo do lábio superior (sto); altura do lábio inferior (sto-gn), que corresponde à distância entre o ponto mais alto do lábio inferior (sto) e o gnátio (gn); altura do filtro (sn-ls), que corresponde à distância entre o ponto subnasal (sn) e o ponto labial superior; terço superior da face (tr-g), que corresponde à medida do trichion (tr) à glabela $(\mathrm{g})$; terço médio da face (g-sn), que corresponde à medida da glabela ( $\mathrm{g}$ ) ao subnasal (sn); terço inferior da face (sn-gn), que corresponde à medida do subnasal (sn) ao gnátio (gn); distância entre o canto externo do olho (ex) e o cheilion (ch), que corresponde à distância entre esses pontos da face $^{(7)}$. Também foram analisadas as proporções orofaciais terço superior da face/terço inferior da face (tr-g/g-sn) e terço médio da face/terço inferior da face ( $\mathrm{g}$-sn/sn-gn). Todas as medidas foram realizadas uma vez.

A análise estatística foi feita no Centro de Métodos Quantitativos da Faculdade de Medicina de Ribeirão Preto (CEMEQ). Foi utilizado o modelo de regressão linear com efeitos mistos (efeitos aleatórios e fixos) ${ }^{(16)}$. Tal modelo, tem como pressuposto, que o resíduo obtido por meio da diferença entre os valores preditos pelo modelo e os valores observados tenha distribuição normal com média 0 e variância constante. O ajuste do modelo foi feito por meio do software SAS, versão 9.0. Ainda, foi utilizado o teste Wilcoxon, que é uma técnica não paramétrica indicada para comparar duas distribuições. Sendo assim, ela não requer suposições quanto à distribuição dos dados. Foram avaliadas também as correlações entre as diferentes medidas realizadas e entre a altura corporal e as medidas. Para tanto foi calculado o coeficiente de correlação de Pearson (r). As análises foram realizadas com o auxílio do software $\mathrm{SAS}^{\circledR}$ 9.0, por meio da PROC IML. Os resultados são apresentados em média, desvio padrão da média, intervalo de confiança 95\%, e o coeficiente de correlação de Pearson (r).

\section{RESULTADOS}

A distribuição das alturas dos participantes está apresentada na Tabela 1.

Tabela 1. Distribuição da altura dos participantes em relação ao gênero

\begin{tabular}{lcccccc}
\hline & \multicolumn{3}{c}{ Altura entre 1,49 e $1,65 \mathrm{~m}$} & \multicolumn{3}{c}{ Altura entre 1,66 e $1,89 \mathrm{~m}$} \\
\cline { 2 - 7 } & Mulheres & Homens & Total & Mulheres & Homens & Total \\
\hline Número & 37 & 4 & 41 & 19 & 40 & $1,79 \pm 0,06$ \\
Altura (média $\pm \mathrm{DP}$ ) & $1,59 \pm 0,05$ & $1,60 \pm 0,06$ & $1,59 \pm 0,05$ & $1,69 \pm 0,04$ & $1,75 \pm 0,06$ \\
\hline
\end{tabular}

Legenda: $\mathrm{DP}=$ desvio-padrão 
Em relação às medidas antropométricas orofaciais foram encontrados maiores valores para os homens em comparação com as mulheres $(\mathrm{p} \leq 0,02)$ (Tabela 2$)$.

Nos indivíduos com altura corporal entre 1,66 e 1,89 m houve diferença entre homens e mulheres, com maiores valores encontrados para os homens $(\mathrm{p}<0,03)$ (Tabela 2$)$. Não houve diferença entre mulheres com altura corporal entre 1,49 e $1,65 \mathrm{~m}(\mathrm{n}=37)$ e em mulheres com altura corporal entre 1,66 e $1,89 \mathrm{~m}(\mathrm{n}=19)(\mathrm{p}>0,05)$ (Tabela 3$)$.

Houve correlação positiva entre a maioria das medidas realizadas. Na Tabela 4 estão apresentadas as medidas nas quais, considerando os 100 indivíduos, o coeficiente de correlação de Pearson (r) foi acima de 0,715 , indicando forte correlação entre elas $(\mathrm{p}<0,001)$.

Não houve correlação (coeficiente de correlação $r$ entre 0 e 0,183$)$ entre as medidas terço médio da face $(\mathrm{g}$-sn): altura do filtro (sn-ls); terço médio da face ( $\mathrm{g}$-sn): altura do lábio inferior (sto-gn); altura do filtro (sn-sto): terço superior da face (tr-g); altura do filtro (sn-sto): altura do lábio inferior (sto-gn); altura do filtro (sn-sto): terço superior da face (tr-g) $(\mathrm{p}>0,07)$ (Tabela 5).
Entre as mulheres não houve correlação entre a altura corporal e as medidas orofaciais, mas entre os homens houve correlação positiva fraca entre altura corporal e as medidas terço médio da face (g-sn), distância entre o canto externo do olho (ex) e o cheilion (ch) esquerdo, e na proporção entre o terço médio e o terço inferior da face $(\mathrm{g}$-sn/sn-gn) $(\mathrm{p}<0,04)$. Com a análise de regressão multivariada foi observado que nos homens a medida do terço médio da face ( $\mathrm{g}$-sn) teve correlação positiva com a altura $(r=0,410, p<0,01)$, sendo as outras correlações com resultados significativos consequentes da medida do terço médio da face (g-sn).

Os indivíduos da raça negra tiveram maiores medidas do terço inferior da face (sn-gn) (negros: homens: 77,8 mm, mulheres: $66,6 \mathrm{~mm}$; brancos: homens: $72,5 \mathrm{~mm}$, mulheres: $65,3 \mathrm{~mm})$, e da distância entre o canto externo do olho (ex) e o cheilion (ch) esquerdo (negros: homens: 73,6 mm, mulheres: $70,1 \mathrm{~mm}$; brancos: homens: $72,7 \mathrm{~mm}$, mulheres: $67,3 \mathrm{~mm})$ do que os indivíduos da raça branca $(\mathrm{p}<0,04)$, com a diferença entre homens e mulheres observada entre os negros e entre os brancos.

Tabela 2. Comparação das medidas antropométricas orofaciais segundo o gênero

\begin{tabular}{|c|c|c|c|c|c|}
\hline \multirow{2}{*}{ Medidas (mm) } & \multicolumn{2}{|c|}{ Mulheres } & \multicolumn{2}{|c|}{ Homens } & \multirow{2}{*}{ Valor de $\mathrm{p}^{*}$} \\
\hline & Média $\pm D P$ & IC95\% & Média $\pm \mathrm{DP}$ & IC95\% & \\
\hline Terço médio da face (g-sn) & $58,7 \pm 5,3$ & $57,3-60,2$ & $61,1 \pm 4,8$ & $59,7-62,6$ & 0,01 \\
\hline Altura do filtro (sn-ls) & $15,3 \pm 2,0$ & $14,8-15,9$ & $17,2 \pm 3,4$ & $16,2-18,2$ & $<0,01$ \\
\hline Altura do lábio superior (sn-sto) & $22,5 \pm 4,2$ & & $24,7 \pm 3,5$ & $23,6-25,8$ & $<0,01$ \\
\hline Altura do lábio inferior (sto-gn) & $43,7 \pm 5,3$ & $42,3-45,1$ & $49,3 \pm 5,1$ & $47,7-50,8$ & $<0,01$ \\
\hline $\begin{array}{l}\text { Distância entre o canto externo do olho (ex) } \\
\text { e o cheilion (ch) esquerdo (ex-ch(E)) }\end{array}$ & $68,2 \pm 3,9$ & $67,2-69,3$ & $72,8 \pm 4,0$ & $71,6-74,0$ & $<0,01$ \\
\hline Terço superior da face/terço inferior da face (tr-g/g-sn) & $58,5 \pm 5,4$ & $57,0-59,9$ & $61,6 \pm 6,0$ & $59,8-63,4$ & 0,01 \\
\hline Terço médio da face/terço inferior da face (g-sn/sn-gn) & $62,9 \pm 4,0$ & $61,3-63,4$ & $67,5 \pm 4,5$ & $66,0-68,8$ & $<0,01$ \\
\hline
\end{tabular}

* O valor de $p$ refere-se à comparação entre homens e mulheres

Legenda: $\mathrm{DP}$ = desvio-padrão; $I \mathrm{C}=$ intervalo de confiança

Tabela 3. Comparação das medidas antropométricas orofaciais segundo o gênero e a altura

\begin{tabular}{|c|c|c|c|c|c|}
\hline \multirow{2}{*}{ Medidas (mm) } & \multicolumn{2}{|c|}{ Altura entre 1,49 e 1,65 m } & \multicolumn{2}{|c|}{ Altura entre 1,66 e $1,89 \mathrm{~m}$} & \multirow{2}{*}{ Valor de $\mathrm{p}$} \\
\hline & Mulheres & Homens & Mulheres & Homens & \\
\hline Terço superior da face (tr-g) & $58,3 \pm 7,4$ & $57,0 \pm 11,8$ & $58,0 \pm 7,2$ & $62,6 \pm 8,8$ & 0,11 \\
\hline Terço médio da face (g-sn) & $58,3 \pm 3,8$ & $55,8 \pm 5,2$ & $59,5 \pm 7,5$ & $61,7 \pm 4,5$ & 0,01 \\
\hline Altura do filtro (sn-ls) & $15,2 \pm 1,9$ & $17,8 \pm 4,0$ & $15,6 \pm 2,4$ & $17,1 \pm 3,4$ & 0,03 \\
\hline Altura do lábio superior (sn-sto) & $22,9 \pm 4,7$ & $24,0 \pm 2,5$ & $21,8 \pm 3,0$ & $24,8 \pm 3,6$ & 0,07 \\
\hline $\begin{array}{l}\text { Distância entre o canto externo do olho (ex) } \\
\text { e o cheilion (ch) esquerdo (ex-ch(E)) }\end{array}$ & $68,1 \pm 4,1$ & $70,5 \pm 4,2$ & $68,4 \pm 3,7$ & $73,1 \pm 3,9$ & $<0,01$ \\
\hline Terço superior da face/terço inferior da face (tr-g/g-sn) & $58,3 \pm 4,8$ & $56,5 \pm 6,6$ & $58,8 \pm 6,5$ & $62,1 \pm 5,7$ & 0,01 \\
\hline Terço médio da face/terço inferior da face (g-sn/sn-gn) & $62,1 \pm 3,8$ & $62,6 \pm 3,6$ & $63,0 \pm 4,2$ & $67,9 \pm 4,3$ & $<0,01$ \\
\hline
\end{tabular}

Média \pm desvio-padrão; $O$ valor de p refere-se à comparação de homens e mulheres com altura entre 1,66 e 1,89 m 
Tabela 4. Medidas antropométricas orofaciais com correlação foi acima de 0,715 (Coeficiente de correlação de Pearson - r), indicando forte correlação entre elas $(p<0,001)$

\begin{tabular}{lcccc}
\hline Medidas & Mulheres & Homens & Total & Valor de $p$ \\
\hline ex-ch E:ex-ch D & 0,753 & 0,854 & 0,830 & $<0,001$ \\
sn-gn:sto-gn & 0,666 & 0,821 & 0,800 & $<0,001$ \\
g-sn:g-sn sn-gn & 0,700 & 0,748 & 0,716 & $<0,001$ \\
g-sn:tr-g g-sn & 0,808 & 0,709 & 0,776 & $<0,001$ \\
g-sn sn-g:sn-g & 0,739 & 0,861 & 0,856 & $<0,001$ \\
tr-g:tr-g g-sn & 0,901 & 0,927 & 0,917 & $<0,001$ \\
\hline
\end{tabular}

Tabela 5. Correlação entre as medidas antropométricas orofaciais e a altura (Coeficiente de correlação de Pearson - r)

\begin{tabular}{|c|c|c|c|c|}
\hline \multirow{2}{*}{ Medidas } & \multicolumn{2}{|c|}{ Mulheres } & \multicolumn{2}{|c|}{ Homens } \\
\hline & $r$ & Valor de $p$ & $r$ & Valor de $p$ \\
\hline Terço superior da face (tr-g) & 0,048 & 0,724 & $-0,032$ & 0,835 \\
\hline Terço médio da face (g-sn) & 0,091 & 0,504 & 0,410 & 0,006 \\
\hline Terço inferior da face (sn-gn) & 0,121 & 0,375 & 0,169 & 0,271 \\
\hline Altura do filtro (sn-ls) & 0,157 & 0,249 & $-0,197$ & 0,199 \\
\hline Altura do lábio superior (sn-sto) & 0,014 & 0,917 & $-0,116$ & 0,452 \\
\hline Altura do lábio inferior (sto-gn) & 0,040 & 0,769 & 0,270 & 0,076 \\
\hline Distância entre o canto externo do olho (ex) e o cheilion (ch) direito (ex-ch(D)) & $-0,003$ & 0,984 & 0,234 & 0,126 \\
\hline Distância entre o canto externo do olho (ex) e o cheilion (ch) esquerdo (ex-ch(E)) & $-0,043$ & 0,754 & 0,322 & 0,033 \\
\hline Terço superior da face/terço inferior da face (tr-g/g-sn) & 0,077 & 0,573 & 0,140 & 0,366 \\
\hline Terço médio da face/terço inferior da face (g-sn/sn-gn) & 0,137 & 0,315 & 0,337 & 0,025 \\
\hline
\end{tabular}

\section{DISCUSSÃO}

Os resultados indicaram tendência a maiores medidas para as faces masculinas, em comparação com as femininas. Esses dados concordam com pesquisas anteriores ${ }^{(9-11)}$. A literatura aponta para diferenças no crescimento facial entre os gêneros, a partir de dez anos de idade ${ }^{(17)}$. Foi observado que mesmo quando homens e mulheres possuíam altura corporal semelhantes houve diferença nas medidas antropométricas orofaciais; e que em mulheres com altura corporal diferentes, acima e abaixo de $1,65 \mathrm{~m}$, os resultados das medidas antropométricas orofaciais foram os mesmos. Os resultados foram consistentes para as mulheres, uma vez que foi pequeno o número de homens avaliados com menos de $1,65 \mathrm{~m}$.

Os resultados encontrados foram semelhantes aos descritos para altura do lábio superior (sn-sto) (mulheres: 17 a $23 \mathrm{~mm}$, homens: 21 a $26 \mathrm{~mm})^{(9,10)}$ e altura do lábio inferior (sto-gn) (mulheres: 38 a $52 \mathrm{~mm}$, homens: 42 a $58 \mathrm{~mm})^{(10)}$.

Não encontramos na literatura estudos que relacionem a altura corporal com o crescimento facial, mas é sabido que este crescimento sofre influência do gênero ${ }^{(17,18)}$.

As diferenças observadas podem explicar o comportamento de homens e mulheres durante a deglutição ${ }^{(1,2,5)}$. Homens deglutem maior volume de água em cada deglutição, e em maior fluxo, do que mulheres ${ }^{(1,2)}$, sendo que as mulheres tem trânsito oral e faringeano mais longo do que os homens ${ }^{(5)}$. Maiores medidas orofaciais também podem justificar a observação de que os homens tem capacidade de tolerar maior volume de água na cavidade oral do que as mulheres ${ }^{(19,20)}$.

Trabalho anterior descreve que a altura corporal não tem influência significativa na deglutição ${ }^{(1)}$, da mesma maneira que não tem influência nas medidas orofaciais, o que reforça a hipótese de que as diferenças descritas na deglutição de homens e mulheres devem ser consequência, ou estão associadas, a diferenças nas medidas orofaciais e não à altura corporal do indivíduo.

Estudo sobre a morfologia e alterações do perfil facial durante o crescimento relata que o dimorfismo sexual começa a ser notado a partir dos 10 a 12 anos, quando o crescimento do gênero masculino, principalmente das medidas lineares, é responsável pela diferenciação facial. Relata também que o perfil mole se torna mais convexo à medida que o oposto ocorre com o perfil esquelético ${ }^{(17)}$.

A principal diferença do dimorfismo sexual é o tamanho e a configuração do nariz, que leva a diferenças colaterais em outras estruturas topográficas da face. O conjunto de todas as diferenças topográficas regionais faz com que a face feminina seja mais achatada, proporcionalmente mais larga e mais delicada, ao contrário da masculina, que parece mais profunda, mais irregular, protuberante e mais grosseira ${ }^{(18)}$.

Ainda, as diferenças encontradas entre as etnias vão de encontro aos achados da literatura ${ }^{(6,8,12,13)}$, com maiores medidas do terço inferior da face em indivíduos negros ${ }^{(13)}$.

Em relação à correlação entre as medidas antropométricas, observamos que entre a maioria delas houve correlação positiva, demonstrando que quando uma medida aumenta, a outra aumenta proporcionalmente. Sendo assim, podemos inferir que essa proporcionalidade entre as medidas gera um padrão facial harmônico e este deve ter interferência nas funções estomatognáticas. Não podemos deixar de considerar que para que esse padrão facial harmônico se desenvolva há influência da oclusão e da musculatura orofacial.

As medidas antropométricas orofaciais podem influenciar, além da deglutição, a mastigação. Foi demonstrado que homens têm força de mordida maior do que as mulheres e que a altura total da face não se correlaciona com força de mordida. 
Dimensões faciais transversas afetam a força de mordida somente em homens, indicando que homens com faces longas tem menor força de mordida que homens com faces normais ${ }^{(21)}$.

As medidas antropométricas orofaciais foram realizadas uma vez em cada indivíduo. Há uma boa correlação entre os resultados obtidos por diferentes profissionais ${ }^{(15)}$, portanto, repetições das medidas realizadas pela mesma pessoa devem encontrar resultados semelhantes.

Houve uma limitação natural na determinação da raça, uma vez que foi realizada apenas pela cor da pele. É o que foi possível fazer em uma população onde a miscigenação é frequente. Foram classificados como da raça negra aqueles que evidentemente eram negros, sendo os demais classificados como brancos.

É proposto que estes resultados tenham importância clínica, visto que é essencial para o exame fonoaudiológico na avaliação, prognóstico, planejamento e acompanhamento terapêutico na motricidade orofacial. Trata-se de um estudo inédito, levando em consideração não apenas as medidas antropométricas, mas individualizando de acordo com gênero, altura e raça.

\section{CONCLUSÃO}

Existem diferenças nas medidas antropométricas orofaciais de homens e mulheres, sendo que os homens apresentam maiores valores quando comparados com as mulheres. Há correlação positiva entre a maioria das medidas antropométricas orofaciais. A altura corporal dos indivíduos parece não influenciar estas medidas. Comparado com indivíduos da raça branca, os indivíduos da raça negra tem maiores medidas do terço inferior da face e da distância entre o canto externo do olho e o cheilion do lado esquerdo.

\section{AGRADECIMENTOS}

Agradecemos o apoio financeiro concedido pela Fundação de Amparo à Pesquisa do Estado de São Paulo (FAPESP) para a realização deste projeto (Processo FAPESP n ${ }^{\circ}$ 08/58805-6).

* WVN contribuiu com a organização do projeto, coleta dos dados, análise dos resultados e redação; RAC colaborou com a organização do projeto $e$ coleta dos dados; ROD contribuiu com a organização do projeto, análise dos resultados e redação

\section{REFERÊNCIAS}

1. Hughes TA, Wiles CM. Clinical measurement of swallowing in health and in neurogenic dysphagia. Q J Med. 1996;89(2):109-16.
2. Alves LMT, Cassiani RA, Santos, CM, Dantas, RO. Gender effect on the clinical measurement of swallowing. Arq Gastroenterol. 2007;44(3):227-9.

3. Dantas RO, Ferriolli E, Souza MAN. Gender effects on esophageal motility. Braz J Med Biol Res. 1998;31(4):539-44.

4. Gluckman A. Sexual dimorphism in human and mammalian biology and pathology. New York: Academic Press; 1981.

5. Dantas RO, Cassiani, RA, Santos CM, Gonzaga GC, Alves, LMT, Mazin, SC. Effect of gender on swallow event duration assessed by videofluoroscopy. Dysphagia. 2008;24(3):280-4.

6. Farkas LG, Katic MJ, Forrest CR, Alt KW, Bagic I, Baltadjiev G, et. al. International anthropometric study of facial morphology in various ethnic groups/races. J Craniofac Surg. 2005;16(4):615-46.

7. Cattoni, DM. O uso do paquímetro na motricidade orofacial: procedimentos de avaliação. Barueri: Pró Fono; 2006.

8. Zhuang A, Landsittel D, Benson S, Roberge R, Shaffer R. Facial anthropometric differences among gender, ethnicity, and age groups. Ann Occup Hyg. 2010;54(4):391-402.

9. Psillakis JM, Lucardi V. Proporções estéticas da face. In: Psillakis JM, Zanini SA, Mélega JM, Costa EA, Cruz RL. Cirurgia craniomaxilofacial: osteotomias estéticas da face. Rio de Janeiro: Medsi; 1987. p. 297-313.

10. Langlade M. Análise da estética. In: Langlade M. Diagnóstico ortodôntico. São Paulo: Santos; 1995. p. 129-60.

11. Sunguino R, Ramos AL, Terada HH, Furquim LZ, Maeda L, Silva Filho OG. Análise facial. Rev. Dent. Press Ortodon. Ortop. Maxilar. 1996;1(1):86-107.

12. Farkas LG, Katic MJ, Forrest CR. Comparison of craniofacial measurements of young adult African-American and North American white males and females. Ann Plast Surg. 2007;59(6):692-8.

13. Parro FM, Toledo MR, Gomes ICD, Marchesan IQ. Diferenças antropométricas entre mulheres brancas e negras após crescimento puberal. Rev CEFAC. 2005;7(4):458-65.

14. Ramires RR, Ferreira LP, Marchesan IQ, Cattoni DM, Silva MAA. Tipologia facial aplicada à fonoaudiologia: revisão de literatura. Rev Soc Bras Fonoaudiolol. 2010;15(1):140-5.

15. Rodrigues FV, Monção FRC, Moreira MBR, Motta AR. Variabilidade na mensuração das medidas orofaciais. Rev Soc Bras Fonoaudiol. 2008;13(4):332-7.

16. Schall R. Estimation in generalized linear models with random effects. Biometrika. 1991;78(4):719-27.

17. Herdy JLA, Nouer PRA, Junqueira JLC, Gumieiro EH. Morfologia e alterações do perfil facial durante o crescimento. Ortodontia, São Paulo. 2002;35(2):109-19.

18. Enlow DH, Hans MG. Forma e padrão da face. In: Enlow, DH; Hans, MG. Noç̃oes básicas sobre crescimento facial. São Paulo: Santos; 2003. p. 130-1.

19. Nascimento WV, Cassiani RA, Dantas RO. Gender effect on oral volume capacity. Dysphagia. 2011 (in press) DOI: 10.1007/s00455-011-9379-4.

20. Lawless HT, Bender S, Oman C, Pelletier C. Gender, age, vessel size, cup vs. straw sipping, and sequence effects on sip volume. Dysphagia. 2003;18(3):196-202.

21. Koç D, Dogan A, Bek B. Effect of gender, facial dimensions, body mass índex and type of functional occlusion on bite force. J App Oral Sci. 2011;19(3):274-9. 